192/COSMO-17

September 5, 2018

\title{
Topological 2-form Gravity in Four Dimensions
}

\author{
HJor Yol Lee ${ }^{\dagger}$ Akika NAKAmichi ${ }^{\star}$ and TAtsura Ueno ${ }^{\diamond}$ \\ $\star \diamond$ Department of Physics, Tokyo Institute of Technology \\ Oh-okayama, Meguro-ku, Tokyo 152, Japan \\ $\dagger$ Tsukuba Institute of Science and Technology \\ Kamitakatsu, Tsuchiura-shi, Ibaraki 300, Japan
}

\begin{abstract}
A kind of topological field theory is proposed as a candidate to describe the global structure of the 2-form Einstein gravity with or without a cosmological constant. Indeed in the former case, we show that a quantum state in the candidate gives an exact solution of the Wheeler-DeWitt equation. The BRST quantization based on the Batalin-Fradkin-Vilkovisky (BFV) formalism is carried out for this topological version of the 2-form Einstein gravity.
\end{abstract}

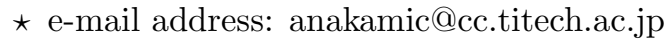

$\diamond$ e-mail address: tatsuya@cc.titech.ac.jp
} 


\section{Introduction}

In recent years, much attention has been focused on the global (topological) aspects of the Einstein gravity. For example, three-dimensional gravity possesses non-trivial global structures both in the classical and quantum levels in spite of the absence of the local fluctuations in the theory. In particular, Witten showed the Einstein action in three dimensions to be exactly equivalent to a Chern-Simons term for the Poincaré group [1]. Using this correspondence, the theory was shown to be exactly soluble and further in [2], the possibility of topology-changing processes was discussed. These advances in three-dimensional gravity suggest that one can also obtain important results by studying the global aspects of the Einstein gravity in four dimensions. However in this case, the presence of the local gravitational waves prevents us from approaching the global feature of the theory directly.

In this paper, we propose a kind of topological field theory (TFT) as a promising candidate which describes the global structure of the four-dimensional Einstein gravity with or without a cosmological constant. This candidate is obtained by modifying an alternative formulation of gravity recently developed by Capovilla, Dell, Jacobson and Meson [3]. This alternative, which we will call the 2-form Einstein gravity, is expressed using anti-self-dual 2-forms as fundamental variables, instead of the metric or the tetrad. In the presence of a cosmological constant, we demonstrate an explicit example that an exact global feature in the Einstein gravity is obtained by examining the TFT in quantum theory. Namely a unique quantum state in the TFT turns out to be one of exact solutions of quantum constraints in the Einstein gravity, which include the Wheeler-DeWitt equation. Moreover, the BRST quantization for the TFT is performed using the Hamiltonian formalism introduced by Batalin, Fradkin and Vilkovisky [4-6]. This powerful method for constrained systems is expected to be useful for further investigations of the quantum feature of gravity. We get the BRST invariant action in covariant form for the TFT with or without a cosmological term. In particular, in the former case, it is shown that the action is identical with the one in Witten's topological 
Yang-Mills theory (TYMT) for the SU(2) group [7-10].

The action of the (Euclidean) 2-form Einstein gravity is given in terms of a symmetric spinor-valued 2-form $\Sigma^{A B}$ and an $\mathrm{SU}(2)$ spin connection 1-form $\omega_{A B}$ in the presence of the cosmological constant $\Lambda$,

$$
S=\int \Sigma^{A B} \wedge R_{A B}-\frac{\Lambda}{24} \Sigma^{A B} \wedge \Sigma_{A B}-\frac{\alpha}{2} \psi_{A B C D} \Sigma^{A B} \wedge \Sigma^{C D},
$$

where $R_{A B} \equiv d \omega_{A B}+\omega_{A C} \wedge \omega^{C}{ }_{B}, \psi_{A B C D}$ is a totally symmetric Lagrange multiplier field and $\alpha$ is an arbitrary parameter. In this formulation, the metric field $g_{\mu \nu}$ is defined in terms of the 2 -form $\Sigma^{A B}$ as

$$
g^{\frac{1}{2}} g_{\mu \nu}=\frac{1}{24} \varepsilon^{\alpha \beta \gamma \delta} \Sigma_{\mu \alpha}^{A B} \Sigma_{\beta \gamma B}^{C} \Sigma_{\delta \nu C A}, \quad g \equiv \operatorname{det}\left(g_{\mu \nu}\right) .
$$

The constraint equations obtained by varying (1) with respect to $\psi_{A B C D}$ imply that $\Sigma^{A B}$ is composed of 1 -form $e^{A A^{\prime}}$ with the $\mathrm{SU}(2)$ spinor and 'primed' spinor indices [3]. Namely,

$$
\Sigma^{(A B} \wedge \Sigma^{C D)}=0 \Longleftrightarrow \Sigma^{A B}=\epsilon_{A^{\prime} B^{\prime}}\left(e^{A A^{\prime}} \wedge e^{B B^{\prime}}\right) .
$$

Using this result and translating the $\mathrm{SU}(2)$ spinor indices into the $\mathrm{SO}(4)$ (the localLorentz) indices [11], we find that (1) is identical with the chiral decomposition of the first order Palatini action in which the usual spin connection is replaced by its anti-self-dual part with respect to the $\mathrm{SO}(4)$ indices. As is discussed in [12], this chiral action also gives the Einstein equation. Since the action (1) describes general relativity, it is invariant under the local-Lorentz transformation and diffeomorphism,

$$
\delta \omega^{A B}=D \theta_{0}^{A B}+\mathcal{L}_{\xi} \omega^{A B}, \quad \delta \Sigma^{A B}=\left[\Sigma, \theta_{0}\right]^{A B}+\mathcal{L}_{\xi} \Sigma^{A B},
$$

where $\mathcal{L}_{\xi}$ is the Lie derivative with respect to a vector field $\xi^{\mu}$, and the local-Lorentz transformation corresponds to the $\mathrm{SU}(2)$ gauge transformation with a parameter $\theta_{0}^{A B}$.

$\sharp 1$ Spinor (and primed spinor) indices are raised and lowered according to the convention in [11]. The symbol $\wedge$ means the exterior product of forms. 


\section{Topological 2-form gravity}

Let us consider the situation when we drop the last term in (1), i.e. $\alpha=0$. From now on we use the internal SU(2) indices $i, j, k, \cdots$ by expanding $\Sigma_{A}{ }^{B}=i \Sigma^{k}\left(\sigma_{k}\right)_{A}{ }^{B}$ and $\omega_{A}^{B}$ in Pauli-matrices $\sigma_{k}$ for simplicity. Then the action (1) with $\alpha=0$ becomes

$$
S_{\alpha=0}=\int \Sigma^{k} \wedge R_{k}-\frac{\Lambda}{24} \Sigma^{k} \wedge \Sigma_{k}
$$

In this particular case, a new symmetry with a parameter 1 -form $\theta_{1}^{k}$ emerges in addition to the local-Lorentz symmetry [3],

$$
\delta \omega^{k}=D \theta_{0}^{k}+\frac{\Lambda}{12} \theta_{1}^{k}, \quad \delta \Sigma^{k}=2\left(\Sigma \times \theta_{0}\right)^{k}+D \theta_{1}^{k}
$$

Although the theory remains invariant under diffeomorphism just as in the $\alpha \neq 0$ case of the previous section, there is no need to add it to (6) because, modulo the equations of motion derived from (5), diffeomorphism with the vector fields $\xi^{\mu}$ can be generated by the combination of the above local-Lorentz and 'new-type' transformations (6) with $\theta_{0}^{k}=\xi^{\nu} \omega_{\nu}^{k}$ and $\theta_{1 \mu}^{k}=2 \xi^{\nu} \Sigma_{\nu \mu}^{k}$. With the appearance of the new-type symmetry, the theory turns out to be on-shell reducible in the sense that the transformation laws (6) are invariant under

$$
\delta \theta_{0}^{k}=-\frac{\Lambda}{12} \epsilon_{0}^{k}, \quad \delta \theta_{1}^{k}=D \epsilon_{0}^{k}
$$

if the equations of motion are satisfied. This means that not all of the parameters in (6) are independent.

For the $\Lambda \neq 0$ case, the action has such a large symmetry under $\delta \omega^{k} \propto \theta_{1}^{k}$ $\left(\delta \Sigma^{k}=D \theta_{1}^{k}\right)$ that the spin connection $\omega^{k}$ can be completely gauged away. This is

\footnotetext{
$\sharp 2 \quad$ We have omitted an overall factor of 2 in (5).

$\sharp 3 \quad$ We use the notation for the SU(2) indices, $F \cdot G \equiv F^{i} G^{i}$ and $(F \times G)^{i} \equiv \varepsilon_{i j k} F^{j} G^{k}$, where $\varepsilon_{i j k}$ is the structure constant of $\mathrm{SU}(2)$.

$D$ is the spin-covariant exterior derivative, e.g. $D \theta_{0}^{k} \equiv d \theta_{0}^{k}+2\left(\omega \times \theta_{0}\right)^{k}$.
} 
very similar to the case of Witten's TYMT for the SU(2) group [7-10]. In fact if one eliminates $\Sigma^{k}$ from the action (5) by using the equations of motion, then the effective action for $\omega^{k}$ reads

$$
S=\frac{6}{\Lambda} \int R^{k} \wedge R_{k}
$$

This is the starting action which leads us to the BRST invariant action of the TYMT in several approaches $[8,10]$. Also for the $\Lambda=0$ case, the action describes a kind of TFT, called BF theory, which has recently been discussed [13-15]. Therefore general relativity, with or without a cosmological constant, is converted into a TFT if the parameter $\alpha$ is chosen to be zero.

In our topological model (5), we expect that the modes of the gravitational wave disappear and there remain no (physical) local fluctuations. To see the change of the physical degrees of freedom and of the symmetries in this process in detail, let us move into the Hamiltonian formalism.

In the topological case $(\alpha=0)$, the action (5) becomes in canonical form,

$$
S=\int d t \int d^{3} x\left[\dot{\omega}_{a} \cdot B^{a}-\omega_{0} \cdot \varphi-\Sigma_{a 0} \cdot \phi^{a}\right] .
$$

The canonical variables are $\omega_{a}^{k}$ and their conjugate momenta $B_{k}^{a} \equiv \varepsilon^{a b c} \Sigma_{b c}^{k}$, which are the spatial components of the spin connection $\omega^{k}$ and the 2-form $\Sigma^{k}$. Varying (9) with respect to their time components $\omega_{0}^{k}$ and $\Sigma_{a 0}^{k}$, we get two sets of constraints,

$$
\varphi_{k} \equiv-D_{a} B_{k}^{a} \approx 0, \quad \phi_{k}^{a} \equiv 2\left(\varepsilon^{a b c} R_{b c}^{k}-\frac{\Lambda}{12} B_{k}^{a}\right) \approx 0
$$

The Poisson brackets among them are given by

$$
\begin{aligned}
& \left\{\varphi_{i}(\mathbf{x}), \varphi_{j}(\mathbf{y})\right\}=-2 \varepsilon_{i j k} \varphi_{k}(\mathbf{x}) \delta^{3}(\mathbf{x}-\mathbf{y}), \quad\left\{\phi_{i}^{a}(\mathbf{x}), \phi_{j}^{b}(\mathbf{y})\right\}=0 \\
& \left\{\varphi_{i}(\mathbf{x}), \phi_{j}^{a}(\mathbf{y})\right\}=-2 \varepsilon_{i j k} \phi_{k}^{a}(\mathbf{x}) \delta^{3}(\mathbf{x}-\mathbf{y})
\end{aligned}
$$

All the constraints are of first class and the algebra is closed. The constraints $\varphi_{k}$ and $\phi_{k}^{a}$ generate the local-Lorentz and new-type transformations in (6) respectively. In this canonical formulation, the on-shell reducibility (7) appears as a 
linear dependence of the constraints,

$$
D_{a} \phi_{k}^{a}-\frac{\Lambda}{6} \varphi_{k}=0
$$

Taking account of this relation, the number of independent constraints is nine at each spatial point, which is just equal to the number of canonical coordinates. Accordingly the physical degrees of freedom for this topological case are $9-9=0$ as expected.

On the other hand, in the Einstein gravity $(\alpha \neq 0)$, we have to solve the constraint equations (3) which can be considered as five linear equations for nine Lagrange multipliers $\Sigma_{a 0}^{k}[3]$. The solution is expressed with four arbitrary variables $N^{a}$ (shift vector) and $\underset{\sim}{N}$ (lapse density of weight -1$)$,

$$
\Sigma_{a 0}^{k}=-\frac{1}{4} \varepsilon_{a b c}\left[N^{b} B_{k}^{c}+\underset{\sim}{N}\left(B^{b} \times B^{c}\right)^{k}\right]
$$

Substituting this result for the canonical action (9), we now have four constraints, together with $\varphi_{k}$ in (10), which are associated with the Lagrange multipliers $N^{a}$ and $\underset{\sim}{N}$

$$
\begin{aligned}
C_{a} & \equiv \frac{1}{4} \varepsilon_{a b c} B^{b} \cdot \phi^{c}=B^{b} \cdot R_{a b} \approx 0 \\
C & \equiv \frac{1}{4} \varepsilon_{a b c}\left(B^{a} \times B^{b}\right) \cdot \phi^{c}=\left(B^{a} \times B^{b}\right) \cdot\left(R_{a b}-\frac{\Lambda}{24} \varepsilon_{a b c} B^{c}\right) \approx 0 .
\end{aligned}
$$

Since the forms of these constraints are just the same as the ones in the Ashtekar formalism $[16,17]$, we can easily identify $\varphi_{k}, C_{a}$ and $C$ with the independent first class constraints corresponding to the generators of the local-Lorentz transformation, spatial diffeomorphism and temporal diffeomorphism respectively. But the constraint algebra is now open as usual in the case of gravity. In this sense the 2-form Einstein gravity is interpreted as a natural covariantization of the Ashtekar formalism. The number of the physical degrees of freedom for the Einstein gravity becomes $9-7=2$, the modes of the gravitational wave. 
As for the relation between the symmetry of the Einstein gravity and that of its topological version, an important observation is that all the constraints in the former are linear combinations of those in the latter. Especially four diffeomorphism generators $C_{a}$ and $C$ in (14) are linearly dependent on nine 'new-type' generators $\phi_{k}^{a}$ in (10). This situation can be understood as follows; the large symmetry of the topological model is partially broken in the Einstein gravity leaving only local-Lorentz and diffeomorphism symmetries intact and as a result the gravitational waves are induced. Note that the transition is caused by recovering the term with the Lagrange multiplier field $\psi_{A B C D}$ in the action (1). With the help of the equations of motion derived from the action (1) with $\alpha \neq 0$, this $\psi_{A B C D}$ is determined to be proportional to the anti-self-dual part of the Weyl (conformal) tensor which just governs the modes of the gravitational wave.

This leads us to an expectation that our topological model is a useful tool to capture the global aspects of the 2-form Einstein gravity. We shall demonstrate an explicit example for such a use in the following. In the Dirac approach for quantization [18], one has to impose quantum conditions to choose physical wave functional $\Psi$. In the topological case with $\Lambda \neq 0$, these conditions can be expressed using the constraints $(10)$ in $\omega_{a}^{k}$ representation,

$$
\begin{aligned}
& \varphi_{k}(\omega, \delta / \delta \omega) \Psi(\omega)=i D_{a}\left(\delta / \delta \omega_{a}^{k}\right) \Psi(\omega)=0, \\
& \phi_{k}^{a}(\omega, \delta / \delta \omega) \Psi(\omega)=2\left(\varepsilon^{a b c} R_{b c}^{k}+i \frac{\Lambda}{12} \delta / \delta \omega_{a}^{k}\right) \Psi(\omega)=0 .
\end{aligned}
$$

We can easily solve these equations to obtain the unique functional of $\omega_{a}^{k}$,

$$
\Psi(\omega)=\exp \left(\frac{6 i}{\Lambda} I_{C-S}\right), \quad I_{C-S} \equiv \int d^{3} x \varepsilon^{a b c} \omega_{a} \cdot\left(\partial_{b} \omega_{c}+\frac{2}{3}\left(\omega_{b} \times \omega_{c}\right)\right)
$$

where $I_{C-S}$ is the Chern-Simons term on the three-dimensional boundary. This type of solution is also found in a different version of topological gravity [15]. The functional $\Psi(\omega)$ can also be considered as the BRST invariant 'vacuum' because it is the unique representative annihilated by the BRST operator defined using (17) below. 
Moreover $\Psi(\omega)$ becomes a special solution of all quantum constraints including the Wheeler-DeWitt equation in the 2-form Einstein gravity if the operator ordering is arranged as in (14). This ordering is adequate for our discussion since it is consistent with the commutation relations among the constraint operators [19]. This $\Psi(\omega)$ is nothing but the Euclidean version of the wave functional discovered by Kodama [20,21]. Therefore the physical space of the topological model is contained in that of the 2-form Einstein gravity.

From this fact, we expect that more informations about the global aspects of the Einstein gravity can be extracted by investigating its topological version further. Henceforth we quantize the topological model with the method introduced by Batalin, Fradkin and Vilkovisky [4-6], which seems to be one of the most suitable procedures to examine the nature of topological quantum field theory (TQFT).

\section{BRST quantization of topological 2-form gravity}

In the classification in $[5,6]$, our system with the action (5) corresponds to a first-stage reducible theory due to the presence of the linearly dependent relation $(12)^{\sharp 4}$ Accordingly we have to enlarge the original phase space of $\left(\omega_{a}^{i}, B_{i}^{a}\right)$ by introducing (i) the Lagrange multipliers and their conjugate momenta; bosonic fields $\left(\lambda^{i}, \pi_{i}\right),\left(\lambda_{a}^{i}, \pi_{i}^{a}\right)$ and fermionic fields $\left(\lambda_{1}^{i}, \pi_{1 i}\right)$, (ii) the conjugate pairs of the fermionic ghosts $\left(C^{i}, \overline{\mathcal{P}}_{i}\right),\left(C_{a}^{i}, \overline{\mathcal{P}}_{i}^{a}\right),\left(\bar{C}_{i}, \mathcal{P}^{i}\right),\left(\bar{C}_{i}^{a}, \mathcal{P}_{a}^{i}\right)$ and (iii) the conjugate pairs of the bosonic 'ghosts for ghosts' $\left(C_{1}^{i}, \overline{\mathcal{P}}_{1 i}\right),\left(\bar{C}_{1 i}, \mathcal{P}_{1}^{i}\right)$. The fields with subscript 1 belong to the class of the first-stage. Besides these fields, we need the conjugate pairs of the 'extraghosts', bosonic $\left({ }^{e x} \lambda^{i},{ }^{e x} \pi_{i}\right)$ and fermionic $\left({ }^{e x} \bar{C}_{i},{ }^{e x} \mathcal{P}^{i}\right)$, in order to make the theory covariant completely. The momenta for the Lagrange multipliers and ${ }^{e x} \pi_{i}$ are constrained to vanish. We assign to the ghosts $C^{i}, C_{a}^{i}$ (the antighosts $\left.\bar{C}_{i}, \bar{C}_{i}^{a}\right)$ the ghost number $g h=1(-1)$ and $g h\left(\lambda_{1}^{i}, C_{1}^{i}, \bar{C}_{1 i},{ }^{e x} \lambda^{i},{ }^{e x} \bar{C}_{i}\right)=$ $(1,2,-2,0,-1)$. The ghost number for each momentum field is set to be opposite

\footnotetext{
$\sharp 4$ In the following, we use the convention in [6] and the notation, $F_{[\mu \nu]} \equiv \frac{1}{2}\left(F_{\mu \nu}-F_{\nu \mu}\right)$.
} 
to its conjugate. Furthermore $\lambda_{1}^{i}, \bar{C}_{i}, \bar{C}_{i}^{a},{ }^{e x} \bar{C}_{i}, \overline{\mathcal{P}}_{i}$ and $\overline{\mathcal{P}}_{i}^{a}$ are pure imaginary while the other fields are real.

Using these fields, the BRST charge is constructed as follows,

$$
\begin{aligned}
& \Omega=\int d^{3} x\left[C \cdot \varphi+C_{a} \cdot \phi^{a}+i C_{1} \cdot\left(D_{a} \overline{\mathcal{P}}^{a}-\frac{\Lambda}{6} \overline{\mathcal{P}}\right)-(C \times C) \cdot \overline{\mathcal{P}}\right. \\
& \left.-2\left(C \times C_{a}\right) \cdot \overline{\mathcal{P}}^{a}+2\left(C_{1} \times C\right) \cdot \overline{\mathcal{P}}_{1}+\pi \cdot \mathcal{P}+\pi^{a} \cdot \mathcal{P}_{a}+\pi_{1} \cdot \mathcal{P}_{1}+{ }^{e x} \pi \cdot{ }^{e x} \mathcal{P}\right]
\end{aligned}
$$

It satisfies the nilpotency condition $\{\Omega, \Omega\}=0$ and is real, fermionic and $g h(\Omega)=$ 1. If setting $\Lambda \neq 0$ and dropping the last extraghost term in (17), this BRST charge becomes equivalent to the one derived in the TYMT [10].

Next we have to choose the gauge fermion $\mathcal{F}$ to be pure imaginary and $g h(\mathcal{F})=$ -1 , in order to obtain the BRST invariant (gauge fixed) action and the BRST transformation in covariant form,

$$
\begin{gathered}
\mathcal{F}=\int d^{3} x\left[\bar{C} \cdot \chi+\bar{C}^{a} \cdot \chi_{a}+\bar{C}_{1} \cdot \chi_{1}+\overline{\mathcal{P}} \cdot \lambda+\overline{\mathcal{P}}^{a} \cdot \lambda_{a}+\overline{\mathcal{P}}_{1} \cdot \lambda_{1}+{ }^{e x} \lambda \cdot \chi^{\prime}\right. \\
\left.+{ }^{e x} \bar{C} \cdot \chi^{\prime \prime}+i \beta \pi_{1} \cdot\left(C_{1} \times \bar{C}_{1}\right)-2 i \gamma\left(\bar{C}_{1} \times C\right) \cdot\left(C_{1} \times \bar{C}_{1}\right)\right]
\end{gathered}
$$

where

$$
\begin{array}{rlrl}
\chi_{i} & \equiv-\partial_{a} \omega_{i}^{a}, & \chi_{a}^{i} \equiv \frac{1}{2} D_{b}\left(\varepsilon^{b}{ }_{a c} B_{i}^{c}\right)-2\left(\lambda \times \lambda_{a}\right)^{i}, \\
\chi_{1 i} \equiv i D_{a} C_{i}^{a}+2\left(\lambda \times \lambda_{1}\right)^{i}, & \chi_{i}^{\prime} \equiv D_{a} \bar{C}_{i}^{a}+2\left(\lambda \times{ }^{e x} \bar{C}\right)^{i}, \\
\chi_{i}^{\prime \prime} \equiv D_{a} \lambda_{i}^{a}, &
\end{array}
$$

and the parameters $\beta, \gamma$ are real. These choices turn out to be the gauge fixing conditions for local-Lorentz, new-type and reducible symmetries respectively in the final covariant expression,

$$
\partial_{\mu} \omega_{i}^{\mu}=0, \quad D_{\nu} \Sigma_{i}^{\mu \nu}=0, \quad D_{\mu} C_{i}^{\mu}=D_{\mu} \bar{C}_{i}^{\mu}=0
$$

\footnotetext{
$\sharp 5$ We use the flat background metric in the gauge fixing procedure for simplicity.
} 
Then the BRST invariant (effective) action $S_{\text {eff }}$ is given by

$$
\begin{aligned}
S_{\text {eff }} & =\int d t\left[\int d ^ { 3 } x \left(\dot{\omega}_{a} \cdot B^{a}+\dot{\lambda} \cdot \pi+\dot{\lambda}_{a} \cdot \pi^{a}+\dot{\lambda}_{1} \cdot \pi_{1}+{ }^{e x} \dot{\lambda} \cdot{ }^{e x} \pi+\dot{C} \cdot \overline{\mathcal{P}}\right.\right. \\
& \left.\left.+\dot{C}_{a} \cdot \overline{\mathcal{P}}^{a}+\dot{C}_{1} \cdot \overline{\mathcal{P}}_{1}+\dot{\bar{C}} \cdot \mathcal{P}+\dot{\bar{C}}^{a} \cdot \mathcal{P}_{a}+\dot{\bar{C}}_{1} \cdot \mathcal{P}_{1}+{ }^{e x} \dot{\bar{C}} \cdot{ }^{e x} \mathcal{P}\right)-H_{e f f}\right]
\end{aligned}
$$

The original Hamiltonian $H_{0}$ vanishes due to the diffeomorphism invariance of the theory and hence the effective Hamiltonian $H_{\text {eff }}$ is equal to $-\{\mathcal{F}, \Omega\}$. After excluding the conjugate momenta for the ghosts and the ghosts for ghosts by using the equations of motion from (21), identifying $\lambda^{i}, \lambda_{a}^{i}, \lambda_{1}^{i}$ and ${ }^{e x} \bar{C}_{i}$ with $\omega_{0}^{i}, \Sigma_{a 0}^{i}, i C_{0}^{i}$ and $\bar{C}_{0}^{i}$, setting the parameters $\beta=\gamma(\equiv \kappa)$ and redefining the following fields,

$$
\begin{array}{ll}
\hat{\pi}_{i}^{a} \equiv \pi_{i}^{a}+2\left(\bar{C}^{a} \times C\right)^{i}, & \hat{\pi}_{i}^{0} \equiv{ }^{e x} \pi_{i}+2\left({ }^{e x} \bar{C} \times C\right)^{i}, \\
\hat{\pi}_{1 i} \equiv \pi_{1 i}-2\left(\bar{C}_{1} \times C\right)^{i}, & \tau^{i} \equiv{ }^{e x} \mathcal{P}^{i}-2\left({ }^{e x} \lambda \times C\right)^{i},
\end{array}
$$

we get

$$
S_{e f f}=S_{c l}+S_{G}+S_{N}+S_{E}
$$

where $S_{c l}$ is the starting action (5) and

$$
\begin{aligned}
S_{G} & =\int d^{4} x\left[\pi \cdot \partial_{\mu} \omega^{\mu}+\partial^{\mu} \bar{C} \cdot\left(D_{\mu} C-\frac{\Lambda}{6} C_{\mu}\right)\right], \\
S_{N} & =\int d^{4} x\left[\hat{\pi}_{\mu} \cdot D_{\nu} \Sigma^{\mu \nu}+2 D^{\mu} \bar{C}^{\nu} \cdot D_{[\mu} C_{\nu]}+\frac{\Lambda}{3}\left(\bar{C}^{\mu} \times C^{\nu}\right) \cdot \Sigma_{\mu \nu}\right. \\
& -i \hat{\pi}_{1} \cdot D_{\mu} C^{\mu}+D^{\mu} \bar{C}_{1} \cdot D_{\mu} C_{1}+\frac{\Lambda}{3} i \bar{C}_{1} \cdot\left(C^{\mu} \times C_{\mu}\right)-\tau \cdot D_{\mu} \bar{C}^{\mu} \\
& \left.-{ }^{e x} \lambda \cdot\left(D_{\mu} \hat{\pi}^{\mu}-\frac{\Lambda}{3}\left(\bar{C}^{\mu} \times C_{\mu}\right)\right)-\frac{i}{2} \varepsilon^{\mu \nu \rho \tau}\left(D_{\mu} \bar{C}_{\nu} \times D_{\rho} \bar{C}_{\tau}\right) \cdot C_{1}\right], \\
S_{E} & =\int d^{4} x\left[-i \kappa\left(\hat{\pi}_{1} \times \hat{\pi}_{1}\right) \cdot C_{1}-\frac{\Lambda}{3} \kappa\left(C_{1} \times \bar{C}_{1}\right)^{2}\right] .
\end{aligned}
$$

All the terms in $S_{N}$ are obtained by the usual Faddeev-Popov procedure with the gauge fixing conditions (20) except for the last cubic ghost term. This non-trivial term stems from the on-shell reducibility (7) of the theory [13]. 
The BRST transformation in covariant form is given by

$$
\begin{array}{ll}
\delta_{B} \omega_{\mu}^{i}=D_{\mu} C^{i}-\frac{\Lambda}{6} C_{\mu}^{i}, \\
\delta_{B} \Sigma_{\mu \nu}^{i}=-2 D_{[\mu} C_{\nu]}^{i}+2\left(\Sigma_{\mu \nu} \times C\right)^{i}+i \varepsilon_{\mu \nu \rho \tau}\left(D^{\rho} \bar{C}^{\tau} \times C_{1}\right)^{i}, \\
\delta_{B} C^{i}=(C \times C)^{i}+\frac{\Lambda}{6} i C_{1}^{i}, \quad \delta_{B} \bar{C}_{i}=-\pi_{i}, \quad \delta_{B} \pi_{i}=0, \\
\delta_{B} C_{\mu}^{i}=2\left(C \times C_{\mu}\right)^{i}+i D_{\mu} C_{1}^{i}, & \delta_{B} C_{1}^{i}=-2\left(C \times C_{1}\right)^{i}, \\
\delta_{B} \bar{C}_{i}^{\mu}=-\hat{\pi}_{i}^{\mu}+2\left(\bar{C}^{\mu} \times C\right)^{i}, & \delta_{B} \hat{\pi}_{i}^{\mu}=2\left(\hat{\pi}^{\mu} \times C\right)^{i}+\frac{\Lambda}{3} i\left(\bar{C}^{\mu} \times C_{1}\right)^{i}, \\
\delta_{B} \bar{C}_{1 i}=\hat{\pi}_{1 i}+2\left(\bar{C}_{1} \times C\right)^{i}, & \delta_{B} \hat{\pi}_{1 i}=2\left(\hat{\pi}_{1} \times C\right)^{i}-\frac{\Lambda}{3} i\left(\bar{C}_{1} \times C_{1}\right)^{i}, \\
\delta_{B}{ }^{e x} \lambda^{i}=\tau^{i}+2\left({ }^{e x} \lambda \times C\right)^{i}, & \delta_{B} \tau^{i}=2(\tau \times C)^{i}-\frac{\Lambda}{3} i\left({ }^{e x} \lambda \times C_{1}\right)^{i} .
\end{array}
$$

The off-shell nilpotency of the transformation is satisfied by all the fields except for the 2 -form $\Sigma_{\mu \nu}^{i}$ and

$$
\delta_{B}^{2} \Sigma_{\mu \nu}^{i}=i \varepsilon_{\mu \nu \tau \rho}\left(\frac{\delta S_{e f f}}{\delta \Sigma_{\rho \tau}} \times C_{1}\right)^{i},
$$

which vanishes by the equations of motion. We have also derived all these results using the antifield formalism introduced by Batalin and Vilkovisky [22].

If we choose the cosmological constant $\Lambda$ and the parameter $\kappa$ in (24) to be zero and drop $S_{G}$ from (23) to keep the local-Lorentz (SU(2)) symmetry, the effective action $S_{\text {eff }}=S_{c l}+S_{N}$ becomes identical with the quantum action discussed in $[13,14]$, which describes a kind of 'Schwarz-type' TQFT.

On the contrary, for the $\Lambda \neq 0$ case, we expect that one can reach the TYMT from our theory because of the equivalence of their starting actions as noted previously. In fact, the quantum action of the TYMT appears if we change the gauge fixing condition for the new-type symmetry as

$$
D_{\nu} \Sigma_{i}^{\mu \nu}=0 \rightarrow D_{\nu}^{+} \Sigma_{i}^{\mu \nu}=0 .
$$

The superscript ' + ' means 'self-dual' for the world indices $\mu, \nu, \cdots$. Besides we 
redefine the following auxiliary and ghost fields,

$$
\pi_{i}^{\mu \nu} \equiv D^{[\mu} \hat{\pi}_{i}^{\nu]}-\frac{\Lambda}{3}\left(C^{[\mu} \times \bar{C}^{\nu]}\right)^{i}, \quad \bar{\chi}_{i}^{\mu \nu} \equiv D^{[\mu} \bar{C}_{i}^{\nu]}
$$

Using the equations of motion derived from the variations with respect to ${ }^{e x} \lambda_{i}, \tau_{i}$ and $\Sigma_{\mu \nu}^{i}$, we get

$$
S_{e f f}=S_{c l}^{\prime}+S_{G}+S_{N}^{\prime}+S_{E}
$$

where $S_{c l}^{\prime}$ is the Pontryagin action (8) and

$$
\begin{aligned}
S_{N}^{\prime}=\int d^{4} x[ & \frac{6}{\Lambda}{ }^{+} \pi_{\rho \tau} \cdot{ }^{+} R^{\rho \tau}+\frac{3}{4 \Lambda}{ }^{+} \pi^{\mu \nu} \cdot{ }^{+} \pi_{\mu \nu}+2^{+} \bar{\chi}^{\mu \nu} \cdot D_{\mu} C_{\nu}-i \hat{\pi}_{1} \cdot D^{\mu} C_{\mu} \\
& \left.+\frac{\Lambda}{3} i \bar{C}_{1} \cdot\left(C^{\mu} \times C_{\mu}\right)-i\left({ }^{+} \bar{\chi}_{\mu \nu} \times{ }^{+} \bar{\chi}^{\mu \nu}\right) \cdot C_{1}+D^{\mu} \bar{C}_{1} \cdot D_{\mu} C_{1}\right] .
\end{aligned}
$$

This $S_{\text {eff }}$ is equivalent to the action of the TYMT [7-10]. Also the elimination of $\Sigma_{\mu \nu}^{i}$ by using the equations of motion brings about the off-shell nilpotent BRST transformation which is the same as the one in the TYMT. We see that the inclusion of the cosmological term changes the 'Schwarz-type' TQFT into the 'Witten-type' TQFT.

\section{Conclusion}

We have clarified the relation between the 2-form Einstein gravity in four dimensions and its topological version, and performed the BRST quantization for the TFT. With the benefit of several technical merits in the TFT, further exploration of the theory will yield fruitful results for the Einstein gravity and is also interesting from the mathematical point of view. Moreover, we can interpret our topological model as an unbroken phase of the Einstein gravity. In fact, we have seen that the partial breakdown of the symmetry in the TFT generates the Einstein gravity. It is sufficiently intriguing for us to pursue the mechanism explaining this process as the spontaneous breakdown of the topological symmetry. 
Acknowledgements: We would like to thank Professors A. Hosoya and N. Sakai for valuable discussion and reading the manuscript. We also acknowledge Drs. M. Abe and K. Amano for useful comments. 


\section{REFERENCES}

1. E. Witten, Nucl. Phys. B311 (1988) 46.

2. E. Witten, Nucl. Phys. B323 (1989) 113.

3. R. Capovilla, J. Dell, T. Jacobson and L. Mason, Class. Quantum Grav. 8 (1991) 41.

4. E.S. Fradkin and G.A. Vilkovisky, Phys. Lett. B55 (1975) 224;

I.A. Batalin and G.A. Vilkovisky, Phys. Lett. B69 (1977) 309.

5. I.A. Batalin and E.S. Fradkin, Phys. Lett. B122 (1982) 157.

6. M. Henneaux, Phys. Rep. 26 (1985) 1.

7. E. Witten, Commun. Math. Phys. 117 (1988) 353.

8. L. Bauliew and I.M. Singer, Nucl. Phys. B (Proc. Suppl.) 5B (1988) 12.

9. J.M.F. Labastida and M. Pernici, Phys. Lett. B212 (1988) 56.

10. Y. Igarashi, H. Imai, S. Kitakado and H. So, Phys. Lett. B227 (1989) 239.

11. R. Penrose and W. Rindler, 'Spinors and Space-time' vol.1 (Cambridge University Press, 1984).

12. T. Jacobson and L. Smolin, Class. Quantum Grav. 5 (1988) 583.

13. M. Blau and G. Thompson, Ann. of Phys. 205 (1991) 130; Phys. Lett. B255 (1991) 535.

14. D. Birmingham, M. Blau, M. Rakowski and G. Thompson, Phys. Rep. 209 (1991) 129.

15. G.T. Horowitz, Commun. Math. Phys. 125 (1989) 417.

16. A. Ashtekar, Phys. Rev. D36 (1987) 1587; 'New Perspectives in Canonical Gravity' (Bibliopolous, Naples, Italy, 1988).

17. A. Ashtekar, J.D. Romano and R.S. Tate, Phys. Rev. D40 (1989) 2572. 
18. P.A.M. Dirac, 'Lectures on Quantum Mechanics', Belfer Graduate School of Science (Yeshiva University, New York, 1964).

19. A. Ashtekar, Phys. Rev. Lett. 57 (1986) 2244.

20. H. Kodama, Phys. Rev. D42 (1990) 2548.

21. H. Ikemori, in 'Proceeding of the Workshop on Quantum Gravity and Topology', edited by I. Oda (Institute for Nuclear Study, University of Tokyo, 1991).

22. I.A. Batalin and G.A. Vilkovisky, Phys. Lett. B102 (1981) 27;

Phys. Rev. D28 (1983) 2567. 